\title{
Green Network Planning Model for Optical Backbones
}

\author{
Jose Gutierrez, Tahir Riaz, Michael Jensen, Jens M. Pedersen, and Ole B. Madsen \\ Department of Electronic Systems, Networking and Security Section, Aalborg University \\ Fredrik Bajers vej 7, 9220 Aalborg Ø, Denmark \\ \{jgl, tahir, mj, jens, obm\} @es. aau.dk
}

\begin{abstract}
Communication networks are becoming more essential for our daily lives and critically important for industry and governments. The intense growth in the backbone traffic implies an increment of the power demands of the transmission systems. This power usage might have a significant negative effect on the environment in general. In network planning there are existing planning models focused on QoS provisioning, investment minimization or combinations of both and other parameters. But for designing green optical backbones there is a lack of a model. This paper presents novel ideas to be able to define an analytical model to consider environmental aspects in the planning stage of backbones design.
\end{abstract}

Keywords: Green network, Network planning, Optical Backbone.

\section{Introduction}

As FTTH is becoming more widely deployed, bandwidth possibilities for users might increase up to Gbs-order connections. This expansion has a significant effect on the backbone traffic. This increment of traffic implies an increment on the power consumption by the whole network system [1-3].

Network planning tools, usually, are focused on economical or performance aspects, but due to this growth of networks, the consideration of environmental aspects might be necessary in a near future.

Currently, there are tools available to be able to analyze the performance or the economical aspects of a network design before its implementation and deployment, examples are [4] and [5]. Geographical Information System (GIS) data is usually used in order to minimize the digging tasks which are directly related to the deployment investment. In addition, the network topologies are defined in such a way that short path distances between nodes (hops) can be established to improve the performance. Unfortunately, the network planning tools have not followed energy efficiency criteria. There is a relation between how a network is designed and implemented and emissions caused, but there is not a solid base to deal with the problem, however many ideas have been proposed lately $[6,7]$.

This work is intended to define the relations between optical backbone network planning and generic Green House Gases emissions. The goal and main contribution is to define an analytical model to be able to relate important parameters used in network planning such as number of nodes, topology, physical length of the network, 
number of users, etc, with GHG emissions. This model has been introduced in [7]. It is possible to study how the optical network infrastructure affects the environment and it might be included as design criteria. Furthermore, a case study is presented as an illustration of the potential model use.

This model is partially based on Life-Cycle Assessment (LCA) [8], a widely used approach to analyze the environmental effects of products or services from "cradleto-grave" and power usage of the different network task and elements, influenced by publications such as [2,3,8-10].

This model lays the first brick towards a more environmental aware network planning, and hopefully it can be used and adapted to find real solutions for this upcoming environmental issue.

The rest of the document is as follows: Section 2 presents important preliminary concepts. Section 3 introduces the model equations and parameters and Section 4 illustrates the practical use of the model. Finally, Section 5 concludes the work.

\section{Preliminary Concepts}

\section{Distributed Weighted Average Distance, $(D W A D)$ :}

It is used to calculate the average number of routers and amplifiers the traffic passes through. It is measured in "hops per bit" and it is dependent on the traffic distribution, a traffic matrix is required.

For the case study, to create the traffic matrix it is assumed that the aggregated traffic by each node is proportional to its population. This traffic is distributed to the rest of the nodes proportionally to their population. Only the internal backbone traffic is considered. For external traffic other transmission concepts such as "node to gateway" should be applied.

The total average distance, $D W A D_{T}$, should consider failures, Eq. (1). $P U_{x}$ is the time percentage a network is at a state of $x$ failures.

To determine $D W A D_{T}$ for the case study, a basic graph analysis script has been implemented. The procedure is to determine the path distance between all the possible pairs of nodes by calculating the corresponding Spanning Tree (ideal scenario, no failures). Then, the process is repeated eliminating the corresponding number of links $([1, f])$ and the average is calculated for each case. The results are deterministic, no simulation involved, in the way that all possible combinations of failure are processed and for all possible pairs of source-destination nodes. This method is more feasible for low $f$ values due to combinational problems.

As a small example, let's consider the possibility of 0 and 1 failures at the same time. The network has no failures $80 \%$ of the time, then $D W A D_{T}=0,8 D W A D_{0}+0,2 D W A D_{1}$. Basic availability concepts are used to calculate the failure probability and down-time of any element [4] and [11].

$$
\mathrm{DWAD}_{\mathrm{T}}=\sum_{\mathrm{x}=0}^{\mathrm{x}=\mathrm{f}} \mathrm{PU}_{\mathrm{x}} \cdot \mathrm{DWAD}_{\mathrm{x}}
$$

Upgradeability: Briefly explained, backbone networks can be implemented following organized interconnection schemes. Networks can be planned to be implemented in stages and at the conclusion of each stage, an organized topology can 
be used for transmissions. In this way, every time a network is upgraded by the addition of new links, the performance consequences can be more predictable. For more information it is recommended to read [12].

$\mathrm{CO}_{2}$ emissions: Even though the final idea in the far future is to be able to produce energy with no emission, "Zero Carbon" concept, the reality is that the trends are going more in the way of the so called "Carbon Neutrality", focused on feasible $\mathrm{CO}_{2}$ reductions [17]. This idea is followed in this document.

Euclidean vs. Real distance: The physical length of the links is calculated as the Euclidean distance between end points. Specialized studies provide ratios EuclideanReal distance of roads [13] and [14]. Thus, their use combined with the Euclidean distances gives an acceptable approximation avoiding the road layout design task.

\section{Notation:}

Emissions: $\mathrm{E}_{\mathrm{NT}}$ are the total emissions of the network, $E D_{x y}, E T_{y}$, and $E M_{x y z}$ are the emissions of deployment, transmission and maintenance where $x$ can be $n, a$ and $l$ for Nodes, Amplifiers and Lines. $y$ corresponds to the topology and it can be $R, H$ and $G$ for Ring, Honeycomb and Grid. In the maintenance case, $z$ can be $M$ for monitoring and $F$ for failure.

Time values: $T D_{x y}, T T_{y}$ and $T M_{y}$ are the periods for deployment, transmission and maintenance. $t D_{x y z}, \mathrm{t} T_{y z}$ and $t M_{x y z}$ are the instant times of each action where $x$ is the element, $y$ is the topology (just as the emissions notation) and $z$ is 0 for the beginning of the period and 1 for the end. $\alpha_{x}, \beta_{x}$ and $\gamma_{x}$ are the emission factors for each of the tasks. $\rho_{\mathrm{x}}, \tau_{\mathrm{x}}$ and $\delta_{\mathrm{x}}$ are their respective decrement rates.

Other variables: TRF traffic, $\mathrm{A}_{\mathrm{A}}$ average amplifiers per link, $\mathrm{A}_{\mathrm{D}}$ average distance and $P U_{f}$ time percentage of a state of $f$ failures. Power consumptions, $P_{n}$ and $P_{a}$ are the power required to treat one bit at a node or amplifier respectively. $P_{l b i t}$ is the average power required to transmit 1 bit from a source to a destination.

\section{The Model}

The goal is to define a model to relate the GHG emissions of a network along its lifetime to the commented parameters in network planning. In this way, it is possible to evaluate the network design options from a new environmental perspective. The model includes some other parameters not strictly related to network planning, i.e emissions per watt generated and not controllable at the design stage. However, the model might help to provide guidelines of how their evolution should be in order to reasonably build environmental backbones. The model can be defined as two simple statements:

- The simplicity of the model allows flexibility of usage.

- The possibility of increasing progressively the complexity of each parameter allows a systematic approach of an efficient match "model-real life". 
Three main stages are considered to contribute to the emissions: Deployment, Transmission and Maintenance. There is the possibility that the power consumption and consequently the potential GHG emissions of one of these actions are not significant. For the definition of the model, everything that might contribute should be included. At the time of obtaining empirical results, maybe some factors will not significantly contribute to the global overview of the network and can be discarded.

Let $E D, E T$ and $E M$ be the emissions caused by the deployment, transmission and maintenance; then the emissions of a network along its lifetime $\left(E_{N T}\right)$ is given by Eq. (2). $X$ can be some other contribution not considered yet, but it leaves the model open for improvement.

$$
E N T=E D+E T+E M+X
$$

\subsection{Deployment Emissions, ED}

They cover the emissions related to the construction of the network and its elements. They can be directly related to the number of elements and length of the links. Similar concept can be found for FTTH (Fiber To The Home) implementation in [15].

There are three types of main affecting elements, number of nodes $N$, number of amplifiers $A$ and network length (including ditches) $L$.

These elements are related to the emissions by defining the $\alpha$ emission factors. Let $\alpha_{n}, \alpha_{a}$ and $\alpha_{l}$ be the emission factors corresponding to the production, transport and installation of the nodes, amplifiers and links equipment given as $\left[\mathrm{CO}_{2}(\mathrm{Kg}) /\right.$ node], $\left[\mathrm{CO}_{2}(\mathrm{Kg}) / \mathrm{amp}\right]$ and $\left[\mathrm{CO}_{2}(\mathrm{Kg}) / \mathrm{km}\right]$. Thus, the format of $E_{D}$ can be defined as Eq. (3).

$$
E D=E D_{n}+E D_{a}+E D_{l}=\alpha_{n} \cdot N+\alpha_{a} \cdot A+\alpha_{l} \cdot L .
$$

In reality, networks are not instantaneously built, it is a time process. The $\alpha$ parameters, will vary in time, i.e the process of producing fibre might involve lower emissions in 10 year. Hence, the variables should be continuous and time dependent. The elements rate of deployment might also be time dependent. Eq. (4) presents $E D$ for a generic deployment time $T D$ when considering time dependent parameters.

$$
E D(T D)=\int_{0}^{T D} \alpha_{n}(t) \cdot N(t)+\alpha_{a}(t) \cdot A(t)+\alpha l(t) \cdot L(t) d t .
$$

The parameters calculations can be as complex as desired; however this work is not focused on getting into their specific details. In fact, some of them will require intense research work before they can be used in reality.

\subsection{Transmission Emissions, ET}

These emissions are related to the transmission of information. Every bit transmitted, routed or amplified at each of the active devices will consume energy [10]. To transmit one bit from any source to a destination is energy consuming and this energy is assumed to involve emissions. Let $P_{n}$ and $P_{a}$ be the power consumption to receive, treat and retransmit 1 bit at a node and amplifier respectively, both given as [W/bit]. Let $A_{D}$ and $A_{A}$ be the path average distance, given as [hops/bit] and average number of amplifiers per link. The calculation of $A_{D}$ is introduced at Section $2(D W A D)$ and it 
can be time dependent due to topology link upgrades for example. $A_{A}$ is presented in Eq. (5). $D_{A}$ is the distance between amplifiers or amplifier-node, currently around 70 $\mathrm{km}$, and it can be time dependent $D_{A}(t)$ implying $A_{A}(t)$. \# Links is the number of links.

$P_{n}$ can depend on the type of signal processing at the nodes, $O x E x O$ or all optical, type of routing, hardware design or even cooling systems. Many concepts might be included on one variable. Its format or complexity is not relevant to generically present the model. But it is important to keep in mind that the accuracy of the model comes from the specific definition of all the parameters for each of the technologies.

Eq. (6) presents the format $P_{1 b i t}$, total average power consumption to transmit one bit from source to destination, including the time dependent format ${ }^{1}$.

$$
\begin{gathered}
A A=\frac{L}{\# \text { Links } \cdot D_{A}} . \\
P_{1 b i t}=P_{n} \cdot(A D+1)+P_{a} \cdot A A \cdot A D \\
P_{1 b i t}(t)=P_{n}(t) \cdot(A D(t)+1)+P_{a}(t) \cdot A A(t) \cdot A D(t)
\end{gathered} .
$$

Eq. (7) presents the format of $E T$, including the time dependent form, where $T R F$ is the traffic aggregated to the network per year, $\beta$ is emissions to generate 1 Watt given as $\left[\mathrm{CO}_{2}(\mathrm{~kg}) / \mathrm{W}\right] . T T=t T_{1}-t T_{0}$ is a generic transmission period.

$$
E T=\beta \cdot T R F \cdot T T \cdot P_{1 b i t} E T(T T)=\int_{t T_{0}}^{t T_{1}} \beta(t) \cdot \operatorname{TRF}(t) \cdot P_{1 b i t}(t) d t .
$$

\subsection{Maintenance Emissions, EM}

These emissions cover all the aspects related to the maintenance of the network. Two types of energy consuming actions are considered: Monitoring and Failure reparation and their corresponding emission variables are $E M_{M}$ and $E M_{F}$, Eq. (8). They can also be presented as time functions similarly to Eq. (4). Like the deployment emissions, three elements are considered: nodes, amplifiers and links. The $E M_{F}$ will be clearly conditioned to the size of the network since the failure rate is proportional to the physical length of the links [11].

The emission factors for monitoring actions are defined as $\gamma_{\mathrm{Mx}}$, given as $\left[\mathrm{CO}_{2}(\mathrm{~kg}) /\right.$ element*year $]$ or $\left[\mathrm{CO}_{2}(\mathrm{~kg}) / \mathrm{km}^{*}\right.$ year $\left.\}\right]$ for the links. The emissions due to failure reparations, $\gamma_{\mathrm{Fx}}$ given as $\left[\mathrm{CO}_{2}(\mathrm{~kg}) /\right.$ element*failure $]$ and $F_{x}$ is the failure rate in [failures/year]. $x$ in the variables notation corresponds to the element, being $n$ for nodes, $a$ for amplifiers and $l$ for links. The rest of the variables are: number of nodes $N$, number of amplifiers $A$ and network length $L$. Both formulas can be expressed, but not presented in the document to avoid repetition, as time functions as previously presented for $E D$ and $E T$.

$$
E M=E M M+E M F=(\gamma M n \cdot N+\gamma M a \cdot A+\gamma M l \cdot L) \cdot T T+(\gamma F n \cdot F n+\gamma F a \cdot F a+\gamma F l \cdot F l) \cdot T T
$$

\footnotetext{
${ }^{1}$ The number of nodes in a path is considered to be $A_{D}+1$ since the source and destination nodes also need energy to process the information.
} 


\section{Case Study}

The following case study intends to illustrate one of the potential uses of the model. It is important to remark that, at this stage, it is not possible to provide final numerical solutions, but it is possible to analytically illustrate the idea. For the presentation of more clear equations several assumptions has been taken. The values of some are symbolic but they do not interfere with the presentation of the use of the model.

\subsection{The Scenario and Cases}

The scenario consists of a set of nodes to be interconnected. Three cases are compared for the same area: to implement a physical Ring, Honeycomb (4X5) or Grid (4x5).

For this purpose, the deployment technique commented in Section 2 is followed. The deployment is planned in stages so at the end of each stage there is an organized topology ready to be used for transmissions. As a result, emission equations are presented for three Cases over the same region to illustrate the influence of the network infrastructure parameters. The three cases are:

- Case A: A Ring topology is deployed and remains as final structure (Stage 1).

- Case B: A Ring topology is deployed and when it is installed the network is ready to be used (Stage 1). In that instant, the Honeycomb upgrade begins and this structure remains for the rest of the network's lifetime (Stage 2).

- Case C: As Case B but when the honeycomb is finished (Stage 2), an upgrade begins to form a Grid that will be the final topology (Stage 3).

The chosen scenario is to connect the 20 most populated metropolitan areas in continental Australia $^{2}$. The shortest physical way to interconnect them is determined for each of the topologies and cases using genetic algorithms ${ }^{3}$ [5]. The complexity of the topology problem is $N P$ since theoretically there are $N$ ! possible solutions. Any search method can be used to solve this type of problems.

\subsection{Assumptions}

Deployment: Starts at $t D_{R 0}=0$.

- The deployment is continuous until the definitive topology is deployed. Thus, for example for Case C: $t D_{R 0}=0, t D_{R I}=t D_{H 0}$ and $t D_{H I}=t D_{G 0}$.

- All the nodes are deployed as soon as possible and at a constant rate. For Case $A$ all nodes will be deployed at the Ring stage, $t D_{N T}=t D_{R l}$. For $B$ and $C$, at the Ring stage 18 nodes are installed and the rest at the Honeycomb stage. If a 20 nodes Ring is implemented, some of its links cannot be used to form a Honeycomb. Therefore, it is not an efficient planning procedure. Links are deployed at a constant rate and amplifiers are installed at the same time.

2 Sydney, Melbourne, Brisbane, Perth, Adelaide, Newcastle, Gold coast, Canberra, Wollongong, Sunshine coast, Bendigo, Geelong, Townsville, Cairns, Orange, Alburya, Darwin, Toowoomba, Ballarat, Shoalhaven. Coordinates and Population taken from [18].

3 The same topology might have different length depending on the case since the optimized topology is the definitive. More information in [12]. 
- The $\alpha$ factors have a constant decreasing rate of $\tau$ per year. $\alpha_{\mathrm{x}}(\mathrm{t})=\alpha_{\mathrm{x} 0} \cdot(1-\tau)^{\mathrm{t}}, \alpha_{\mathrm{x} 0}$ is the initial value at $t=0=t D_{R 0}$. There is a need to define a reference value, $\alpha_{\text {xref, }}$ corresponding to the emissions of implementing one node (amplifier or km of link) in one year. In this way $\alpha_{x 0}=\alpha_{x r e f} / T D . x$ corresponds to the element $(n, a$ or $l)$.

$-\mathrm{D}_{\mathrm{A}}=70 \mathrm{~km}$ and it is kept constant (distance between amplifiers).

Transmission: Starts at $t T_{R 0}=t D_{R l}$.

-The topology is not operable until the installation/upgrade is completed.

-The $\beta$ factor have a constant decreasing rate $\rho$ per year. $\beta_{x}(t)=\beta_{x 0} \cdot(1-\rho)^{t}, \beta_{x 0}$ is the initial value at $t=0=t D_{R 0}$.

-The average distance is calculated as $D W A D$, Eq. (1) and up to one failure at the same time. It is assumed shortest path routing and all the links have a constant equal weight when calculating the Spanning Tree in both cases, of 0 and 1 failures.

-Traffic transactions increase rate is kept constant, $T R F_{0} \cdot\left(1+\rho_{T R F}\right)^{t}$.

- $P_{n}$ and $P_{a}$ are assumed to be always constant.

Maintenance: Starts at $t M_{R O}=t D_{R I}$.

-The $\gamma$ factors have a constant decreasing rate $\delta$ per year. $\gamma_{x}(t)=\gamma_{x 0^{*}}(1-\delta)^{t}, \gamma_{\mathrm{x} 0}$ is the initial value at $t=0=t D_{R O}$.

-It is assumed that only link failures are significant to be included in the formulas to illustrate the example. Equipment at the nodes and amplifiers are highly reliable and very unlikely to fail [5]. However, human errors might be included in future.

-The values to calculate the failure rates and element down-time are taken from [4] and [11] and correspond to $M T T F=500 \mathrm{FIT}$ and $M T F=14,4 \mathrm{~h}$.

\subsection{Analysis}

For the rest of the case study, to indicate the case, a superscript (A, B or C) is added to each of the terms, and for example $t D_{R 0}{ }^{A}$ is the beginning of deployment of the Ring at case A. Table 1 presents the equations to be solved to determine the total emissions of Case A. These formulas act as a guideline and, to avoid repetition, for Cases $\mathrm{B}$ and $\mathrm{C}$ they are not presented. The concept is similar but considering the proper time periods, stages and parameters for the different tasks.

Table 1. Case A equations

\begin{tabular}{|c|c|}
\hline Emissions & Case A \\
\hline$E D_{R}\left(T D_{R}^{A}\right)$ & $\int_{0}^{t D_{R_{1}}^{A}} \alpha_{n_{0}} \cdot N \cdot\left(1-\tau_{\alpha_{n}}\right)^{t}+\alpha_{a_{0}} \cdot A \cdot\left(1-\tau_{\alpha_{a}}\right)^{t}+$ \\
\hline$E T_{R}\left(T T_{R}^{A}\right)$ & $\alpha_{l_{0}}^{A} \cdot L \cdot\left(1-\tau_{\alpha_{t}}\right)^{t} d t$ \\
\hline & $\beta_{0} \cdot T R F_{0} \cdot\left(P_{n} \cdot\left(A_{D}+1\right)+P_{a} \cdot A_{D} \cdot A_{A}\right) \cdot$ \\
& $\int_{t T_{R_{0}}}^{t T_{1}^{A}}\left(1-\rho_{B}\right)^{t} \cdot\left(1+\rho_{T R F}\right)^{t} d t$ \\
\hline$E M_{R}\left(T M_{R}^{A}\right)$ & $\int_{t M_{R_{0}}^{A}}^{t}\left(\gamma_{M n_{0}}^{\lambda} \cdot N \cdot\left(1-\delta_{\gamma_{M n}}\right)^{t}+\gamma_{M a_{0}} \cdot A \cdot\left(1-\delta_{\gamma_{M a}}\right)^{t}+\right.$ \\
& $\left.\gamma_{M l_{0}} \cdot L \cdot\left(1-\delta_{\gamma_{M}}\right)^{t}\right) d t$ \\
\hline
\end{tabular}

Fig. 1 presents the planning solutions for the considered cases including time lines illustrating the emissions periods. Some of the time parameters are indicated as 


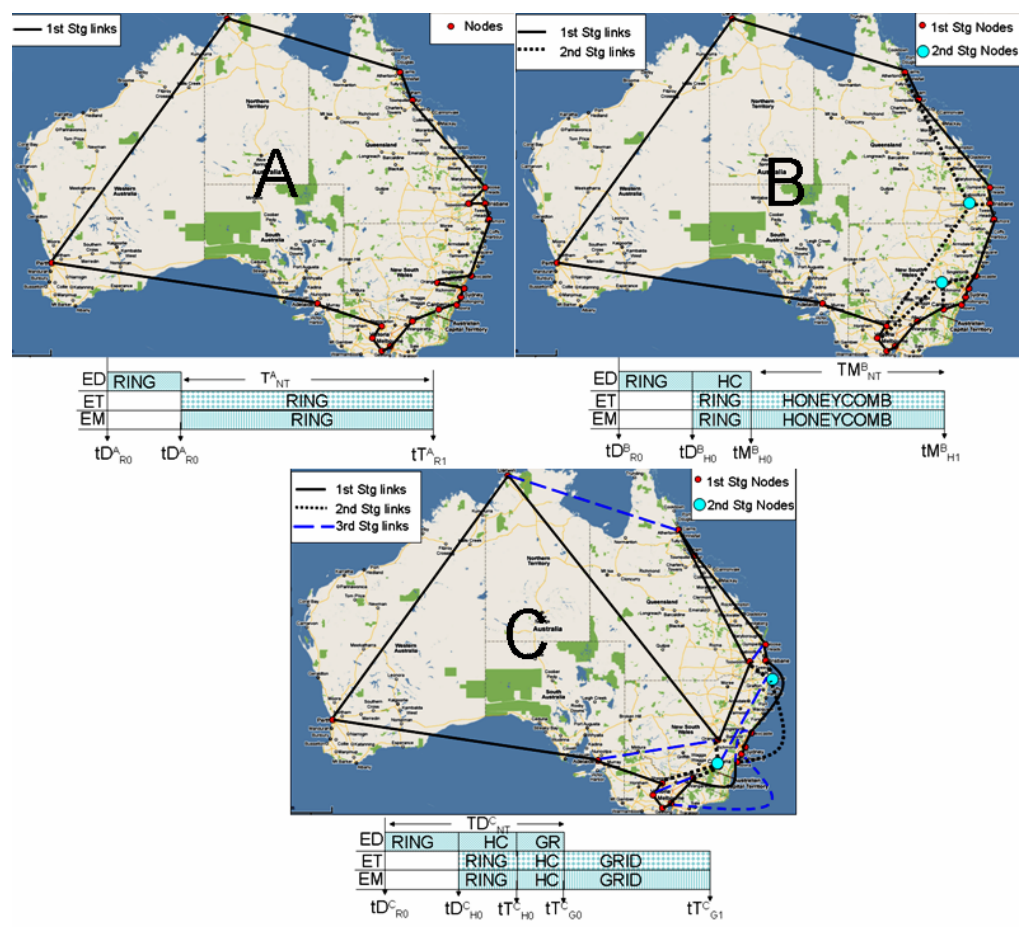

Fig. 1. Case A, B and C

examples for a better understanding. Table 2 presents the numerical values of the planning parameters. The information is divided by Case and Stage displaying for each the corresponding topology and all its parameters (nodes, length, amps ...). The variables $N D p, A D p$ and $L D p$ correspond to the nodes, amplifiers and $\mathrm{km}$ of link to be deployed at each stage. All the values are calculated, there are no assumptions. The information is displayed by case and stage. The information required in order to make these calculations is the following: City coordinates and population, MTTF and MFT, a graph analysis script to calculate the average distance as $D W A D$, as mentioned in Section 2 and a ratio of Euclidean-Real distance of $\sqrt{ } 2$.

Finally, Table 3 presents the resulting equations. In order to provide reasonably short terms, all the $\tau, \rho$ and $\delta$ parameters are given a value of 0,05 (except for $\rho_{\mathrm{TRF}}$ ). This implies a decrement of $5 \%$ per year. $\rho_{\mathrm{TRF}}=0,41$, in the way that traffic volume is doubled every two years as predicted for a near future [16].

The equations are displayed by case, type and period, the total emissions of each case being the sum of all their emissions. The effect of each of the planning factors can clearly be identified in Table 2 . In Table 3, these factors are indicated only in the first equation of each type for clearness of display, but for the rest it is similar.

One of the basic illustrated ideas is that the Grid network contributes more to $E D$ and $E M$ but less to ET than the Honeycomb or Ring. Depending on the values assigned to the stages periods, the emission factors or traffic trends, different options can be evaluated to find the most environmental. 
Table 2. Planning results for cases A, B and C

\begin{tabular}{|c|c|c|c|c|c|c|}
\hline Case & A & \multicolumn{2}{|c|}{ B } & \multicolumn{3}{c|}{ C } \\
\hline Stage & st1 & st1 & st2 & st1 & st2 & st3 \\
\hline Top. & R & R & H & R & H & G \\
\hline Nodes $(N D p)$ & $20(20)$ & $18(18)$ & $20(2)$ & $18(18)$ & $20(2)$ & $20(0)$ \\
\hline Links & 20 & 18 & 24 & 18 & 24 & 31 \\
\hline $\begin{array}{c}\text { Length }[\mathrm{km}] \\
(L D p)\end{array}$ & 14870 & 14344 & 19800 & 20009 & 21684 & 28750 \\
\hline Amplifiers $(A D p)$ & $213(213)$ & $205(205)$ & $283(78)$ & $286(286)$ & $310(24)$ & $411(101)$ \\
\hline$A_{A}$ & 10,55 & 11,40 & 11,79 & 15,89 & 12,92 & 13,26 \\
\hline$F_{L}($ failures year $)$ & 65,13 & 62,83 & 86,72 & 87,64 & 94,98 & 125,92 \\
\hline$P U_{0}$ & 89,3 & 89,7 & 85,7 & 85,6 & 84,4 & 79,3 \\
\hline$D W A D_{0}$ & 5,28 & 4,73 & 3,81 & 4,8 & 3,69 & 3,27 \\
\hline$D W A D_{1}$ & 6,58 & 7,35 & 3,92 & 6,1 & 3,75 & 3,32 \\
\hline$D W A D_{T}$ & 5,42 & 4,99 & 3,83 & 4,98 & 3,70 & 3,28 \\
\hline
\end{tabular}

The model and its solutions combined with some parameters analysis might contribute to some of the following ideas:

-Simply, which, among a set of options, implies the least emissions according to the expected trends of the emission factors or traffic growth.

- How the parameters evolution has to be so Case X is better than Case Y.

- How and when to upgrade a network to make it more environmentally efficient.

- Network planning has always been a matter of considering global optimization between different network properties to find a balance. The addition of the environmental aspect will not change this concept. In fact, the combination of different backbone models (i.e. performance, economical and environmental) can provide a better systematic planning procedure in future.

The three different cases show how the network planning parameters affect each type of emissions. However, it is possible to go a little further in the solution in order to illustrate more clear results; numerical values are given to the time periods. This way it is possible to compare the three solutions just as a function of the emissions factors. Obviously this value depends on the investment for the deployment, high investment more man power and machinery available, so the value is an example. Let the deployment speed be $3000 \mathrm{Km}$ per year ${ }^{4}$ and the corresponding nodes and amplifiers are implemented within the links deployment time. The network has an estimated lifetime of 40 years. Table 4 presents the resulting time periods $(T)$ and instants $(t)$ for these assumptions.

Finally, substituting all the numerical values, Table 5 gives the final results to be compared as a function of the emissions factors, these factors are constant and equal in the three cases. Briefly, the conclusion of this example verifies the affirmations previously stated and in [7].

In terms of deployment and maintenance, the physical length of the links significantly affects the emissions. To deploy and maintain a Ring causes considerably less emissions than a Grid. On the other hand, the reduction of the physical hops of the paths implies a reduction on the ET related to both amplifiers and

\footnotetext{
${ }^{4}$ In this way any of the cases, the deployment can be finalized in 10 years maximum.
} 
Table 3. Cases' practical planning results

\begin{tabular}{|c|c|}
\hline Case & ED \\
\hline $\mathrm{A}, T D_{R}$ & $(\underbrace{20}_{N D_{p}} \alpha_{n r e f}+\underbrace{213}_{A D_{p}} \alpha_{a r e f}+\underbrace{14870}_{L D_{p}} \alpha_{l r e f})\left(1-0,95^{t D_{R 1}^{A}}\right) /\left(0,05 \cdot T D_{R}^{A}\right)$ \\
\hline $\mathrm{B}, T D_{R}$ & $\left(18 \alpha_{n r e f}+205 \alpha_{\text {aref }}+14344 \alpha_{\text {lref }}\right)\left(1-0,95^{t D_{R 1}^{B}}\right) /\left(0,05 \cdot T D_{R}^{B}\right)$ \\
\hline $\mathrm{B}, T D_{H}$ & $\left(2 \alpha_{\text {nref }}+78 \alpha_{\text {aref }}+5456 \alpha_{\text {lref }}\right)\left(0,95^{t D_{H 0}^{H}}-0,95^{t D_{H 1}^{H}}\right) /\left(0,05 \cdot T D_{H}^{B}\right)$ \\
\hline $\mathrm{C}, T D_{R}$ & $\left(18 \alpha_{n r e f}+286 \alpha_{\text {aref }}+20009 \alpha_{l \text { lref }}\right)\left(1-0,95^{t D_{R 1}^{C}}\right) /\left(0,05 \cdot T D_{R}^{C}\right)$ \\
\hline $\mathrm{C}, T D_{H}$ & $\left(2 \alpha_{\text {nref }}+24 \alpha_{\text {aref }}+1675 \alpha_{\text {lref }}\right)\left(0,95^{t D_{H 0}^{C}}-0,95^{t D_{H 1}^{C}}\right) /\left(0,05 \cdot T D_{H}^{C}\right)$ \\
\hline \multirow[t]{2}{*}{$\mathrm{C}, T D_{G}}$, & $\left(101 \alpha_{\text {aref }}+7066 \alpha_{\text {lref }}\right)\left(0,95^{t D_{G}^{C}}-0,95^{t D_{11}^{C}}\right) /\left(0,05 \cdot T D_{G}^{C}\right)$ \\
\hline & ET \\
\hline $\mathrm{A}, T D_{R}$ & $\beta_{0} \cdot T R F_{0} \cdot(\underbrace{6,42}_{D W A D_{T}+1} P_{n}+\underbrace{57,18}_{A_{A}=D W A D_{T}} P_{a})\left((1,33)^{t T_{R 1}^{A}}-(1,33)^{t T_{R 0}^{A}}\right) / 0,28$ \\
\hline $\mathrm{B}, T T_{R}$ & $\beta_{0} \cdot T R F_{0} \cdot\left(5,99 P_{n}+56,89 P_{a}\right)\left((1,33)^{t T R 1}-(1,33)^{t T_{R 0}^{B}}\right) / 0,28$ \\
\hline $\mathrm{B}, T T_{H}$ & $\beta_{0} \cdot T R F_{0} \cdot\left(4,83 P_{n}+45,16 P_{a}\right)\left((1,33)^{t T H 1}-(1,33)^{t T_{H 0}^{B}}\right) / 0,28$ \\
\hline $\mathrm{C}, T T_{R}$ & $\beta_{0} \cdot T R F_{0} \cdot\left(5,98 P_{n}+79,13 P_{a}\right)\left((1,33)^{t T_{R 1}^{C}}-(1,33)^{t T_{R 0}^{C}}\right) / 0,28$ \\
\hline $\mathrm{C}, T T_{H}$ & $\beta_{0} \cdot T R F_{0} \cdot\left(4,70 P_{n}+47,80 P_{a}\right)\left((1,33)^{t T H 1}-(1,33)^{t T_{H 0}^{C}}\right) / 0,28$ \\
\hline $\mathrm{C}, T T_{G}$ & $\beta_{0} \cdot T R F_{0} \cdot\left(4,28 P_{n}+43,49 P_{a}\right)\left((1,33)^{t T_{G 1}^{C}}-(1,33)^{t T_{G}^{C}}\right) / 0,28$ \\
\hline & EM \\
\hline $\mathrm{A}, T M_{R}$ & $(\underbrace{20}_{N} \gamma_{M n_{0}}+\underbrace{213}_{A} \gamma_{M a_{0}}+\underbrace{14870}_{L} \gamma_{M l_{0}}+\underbrace{65,15}_{F_{L}} \gamma_{F l_{0}})\left(0,95^{t M_{R 0}^{\lambda}}-0,95^{t M_{R 1}^{\lambda}}\right) / 0,05$ \\
\hline $\mathrm{B}, T M_{R}$ & $\left(18 \gamma_{M n_{0}}+205 \gamma_{M a_{0}}+14344 \gamma_{M l_{0}}+62,83 \gamma_{F l_{0}}\right)\left(0,95^{t M_{R 0}^{H}}-0,95^{t M_{R 1}^{B}}\right) / 0,05$ \\
\hline $\mathrm{B}, T M_{H}$ & $\left(20 \gamma_{M n_{0}}+283 \gamma_{M a_{0}}+19800 \gamma_{M l_{0}}+86,72 \gamma_{F l_{0}}\right)\left(0,95^{t M_{H 0}^{H}}-0,95^{t M_{H 1}^{H}}\right) / 0,05$ \\
\hline $\mathrm{C}, T M_{R}$ & $\left(18 \gamma_{M n_{0}}+286 \gamma_{M a_{0}}+20009 \gamma_{M l_{0}}+87,64 \gamma_{F t_{0}}\right)\left(0,95^{t M_{R 0}^{C}}-0,95^{t M_{R 1}^{C}}\right) / 0,05$ \\
\hline $\mathrm{C}, T M_{H}$ & $\left(20 \gamma_{M n_{0}}+310 \gamma_{M a_{0}}+21684 \gamma_{M l_{0}}+94,98 \gamma_{F l_{0}}\right)\left(0,95^{t M_{H 0}^{C}}-0,95^{t M_{H 1}^{C}}\right) / 0,05$ \\
\hline $\mathrm{C}, T M_{G}$ & $\left(20 \gamma_{M n_{0}}+411 \gamma_{M a_{0}}+28750 \gamma_{M l_{0}}+125,92 \gamma_{F l_{0}}\right)\left(0,95^{t M_{G 0}^{C}}-0,95^{t M_{G 1}^{C}}\right) / 0,05$ \\
\hline
\end{tabular}

Table 4. Planning results for cases A, B and C

\begin{tabular}{|c|c|c|c|}
\hline T variables & Years & $\mathrm{t}$ variables & Instant (years) \\
\hline$T D_{R}^{A}$ & 5 & $t D_{R 0}^{A}=t D_{R 0}^{B}=t D_{R 0}^{C}$ & 0 \\
\hline$T D_{R}^{B}$ & 4,8 & $t D_{R 1}^{A}=t T_{R 0}^{A}=t M_{R 0}^{A}$ & 5 \\
\hline$T D_{H}^{B}$ & 1,8 & $t D_{R 1}^{B}=t T_{R 0}^{B}=t M_{R 0}^{B}$ & 4,8 \\
\hline$T D_{R}^{C}$ & 6,7 & $t D_{H 1}^{B}=t T_{H 0}^{B}=t M_{H 0}^{B}$ & 6,6 \\
\hline$T D_{H}^{C}$ & 0,5 & $t D_{R 1}^{C}=t T_{R 1}^{C}=t M_{R 0}^{C}$ & 6,7 \\
\hline$T D_{G}^{C}$ & 2,4 & $t D_{H 1}^{C}=t T_{H 1}^{C}=t M_{H 0}^{C}$ & 7,2 \\
\hline$T_{N T}^{C}$ & 40 & $t D_{G 1}^{C}=t T_{G 1}^{C}=t M_{G 0}^{C}$ & 9,6 \\
\hline
\end{tabular}

Table 5. Emissions comparison

\begin{tabular}{|c|c|}
\hline Emission(Case $)$ & Values \\
\hline$E D(A)$ & $18,1 \alpha_{\text {nref }}+192,7 \alpha_{\text {aref }}+13455 \alpha_{\text {lref }}$ \\
\hline$E D(B)$ & $17,9 \alpha_{\text {nref }}+246,2 \alpha_{\text {aref }}+17223 \alpha_{\text {lref }}$ \\
\hline$E D(C)$ & $17,1 \alpha_{\text {nref }}+332,9 \alpha_{\text {aref }}+23288 \alpha_{\text {lref }}$ \\
\hline$E T(A)$ & $\beta_{0} \cdot T R F_{0} \cdot\left(2,06 \cdot 10^{6} P_{n}+1,86 \cdot 10^{7} P_{a}\right)$ \\
\hline$E T(B)$ & $\beta_{0} \cdot T R F_{0} \cdot\left(1,55 \cdot 10^{6} P_{n}+1,45 \cdot 10^{7} P_{a}\right)$ \\
\hline$E T(C)$ & $\beta_{0} \cdot T R F_{0} \cdot\left(1,37 \cdot 10^{6} P_{n}+1,40 \cdot 10^{7} P_{a}\right)$ \\
\hline$E M(A)$ & $258,1 \gamma_{M n_{0}}+2749 \gamma_{M a_{0}}+1,85 \cdot 10^{5} \gamma_{M l_{0}}+840,5 \gamma_{M F l_{0}}$ \\
\hline$E M(B)$ & $258,5 \gamma_{M n_{0}}+3590 \gamma_{M a_{0}}+2,51 \cdot 10^{5} \gamma_{M l_{0}}+1100 \gamma_{M F l_{0}}$ \\
\hline$E M(C)$ & $231,5 \gamma_{M n_{0}}+4566 \gamma_{M a_{0}}+3,19 \cdot 10^{5} \gamma_{M l_{0}}+1399 \gamma_{M F l_{0}}$ \\
\hline
\end{tabular}


nodes. The optimal design decision depends on the numerical values of these emission factors, which with the cooperation of other science disciplines, would be possible in future. In any case, this example can clearly illustrate the basics of the model and how to use and interpret it. The model can be implemented as an evaluation application and sweeps of several of the present variables in the model can be performed to observe the potential contributions depending on the planning decisions, future technological evolutions or even environmental legislation.

\section{Conclusion}

The main contribution is the presentation of a network planning model focused on the emissions generated along the lifetime of an optical backbone. This model relates the classical network planning parameters such as number of nodes, distances between pairs of nodes or physical network length to the emissions generated by the different elements. Three main types of emissions are defined based on Deployment, Transmission and Maintenance actions. The model can be defined as simple as desired but it provides the possibility of extending each of its elements to significant complexity levels. Several parameters present on the model are not strictly related to networks, i.e. emissions per watt generated, thus for the evaluation of environmental planning aspects of backbones, there are many different science fields involved and interrelated. Furthermore, a case study illustrates the potential use and analysis of the model in real networks. Several assumptions had to be made in order to provide solutions equations. It is possible to identify how each of the mentioned network planning parameters (Table 2) affects the emission levels.

The model is defined and presented, and even though it is not possible yet to provide final numerical solutions, the first step has been taken towards the inclusion of environmental aspects in optical network planning and design.

Further research on each of the parameters present in the equations is required to estimate their behavior in time. Each of the emissions factors can be extended or modified to be used for other environmental measurements such as toxicity. Maybe other factors can be included as well such as equipment replacement or recycling for $E M$. In general, there is potential new research on this model.

\section{References}

1. Chabarek, J., Sommers, J., Barford, P., Estan, C., Tsiang, D., Wright, S.: Power Awareness in Network Design and Routing Export. In: INFOCOM 2008 (2008)

2. Chiaraviglio, L., Mellia, M., Neri, F.: Reducing Power Consumption in Backbone Networks. In: ICC 2009, Dresden, Germany (June 2009)

3. Chiaraviglio, L., Mellia, M., Neri, F.: Energy-aware Backbone Networks: a Case Study. In: Workshop on Green Communications, ICC 2009 (2009)

4. To, M., Neusy, P.: Unavailability Analysis of Long-Haul Networks. IEEE Journal on Selected Areas in Communications 12(1) (January 1994)

5. Gutierrez, J., Imine, M., Madsen, O.: Network Planning Using GA For Regular Topologies. In: ICC 2008, Beijing, China (May 2008) 
6. Nielsen, R.H., Mihovska, A., Madsen, O.B.: Energy-efficient deployment through optimizations in the planning of ICT networks. In: WPMC 2009, Japan (2009)

7. Gutierrez, J., Riaz, T., Pedersen, J.M., Madsen, O.B.: A Survey On Environmental Backbone Design and Implementation. In: ISABEL'09, Bratislava, Slovakia (2009)

8. Owens, W.: Life-cycle assessment: Constraints on moving from inventory to impact assessment. Journal of Industrial Ecology 1(1), 37-49 (1997)

9. Landman, P.: High-level power estimation. In: International Symposium on Low Power Electronics and Design, Monterey, CA, USA, August 12-14 (1996)

10. Idzikowski, F.: Power consumption of network elements in IP over WDM networks. TKN Technical Report TKN-09-006, Berlin (July 2009)

11. Challita, A., Tzanakaki, A., Tomkos, I.: Reliability Based Routing in WDM Optical Networks. In: ICTON 2005 (Mo.B1.5). IEEE, Los Alamitos (2005)

12. Gutierrez, J., Riaz, T., Pedersen, J., Madsen, O.: Upgradeability and Predictability Analysis for Mesh Topologies in Optical Distribution Networks. In: WOCN 2009, Egypt (2009)

13. Fernandez, J., Fernandez, P., Pelegrin, B.: Estimating actual distances by norm functions: a comparison between the $1_{k, p, \theta-}$ norm and the $1_{b 1, b 2, \theta}$-norm and a study about the selection of the data set. Comput. Oper. Res. 29(6), 609-623 (2002)

14. Love, R.F., Morris, J.G.: Mathematical models of road travel distances. Management Science 25, 130-139 (1979)

15. Ecobilan for FTTH Council Europe, FTTH Solutions for a Sustainable Development (February 2008), http: / /www. ftthcouncil.eu/

16. Coffman, K.G., Coffman, K.G.: Internet growth: Is there a "Moore's Law" for data traffic. Handbook of Massive Data Sets, 47-93 (2001)

17. Vandenbergh, M.P.: The Carbon-Neutral Individual, vol. 82. University Law Review, New York (2007); Vanderbilt Law and Economics Research Paper No. 07-29

18. http://www.tageo.com/index-e-as-cities-AU.htm 